\title{
Plasma cell pleocytosis in HSV-2 aseptic meningitis
}

\author{
Rohit Kalia $^{1}$, Karen Hennessey ${ }^{2}$ \\ ${ }^{1}$ Internal Medicine Resident, Lincoln Hospital, New York, USA \\ ${ }^{2}$ Chief Department of Infectious Diseases, Lincoln Hospital, New York, USA \\ Email: doctor.kalia@yahoo.com
}

Received 31 August 2013; revised 30 September 2013; accepted 7 October 2013

Copyright (C 2013 Rohit Kalia, Karen Hennessey. This is an open access article distributed under the Creative Commons Attribution License, which permits unrestricted use, distribution, and reproduction in any medium, provided the original work is properly cited.

\begin{abstract}
Plasma cell pleocytosis has been seen in cerebrospinal fluid (CSF) analysis of patients with multiple myeloma, multiple sclerosis, leukemia, west nile encephalitis and lymphoma. There also have been some anecdotal reports suggesting the presence of plasma cells in CSF as possible early indicator of WNV encephalitis. We describe a case of plasma cell pleocytosis in CSF due to HSV-2 meningitis, adding it to the list of possible etiologies for the same.
\end{abstract}

Keywords: HSV Meningitis; Plasma Cell Pleocytosis; Plasma Cells; HSV-2; Herpes Simplex Virus 2

\section{INTRODUCTION}

Aseptic meningitis refers to patients who have clinical and laboratory evidence for meningeal inflammation with negative routine bacterial cultures. Etiologies include enteroviruses, mycobacteria, fungi, spirochetes, parameningeal infections, medications, and malignancy. The majority of cases of aseptic meningitis are caused by enteroviruses, mostly occurring during summer or fall. A review showed that the total cost of an episode aseptic meningitis was from 450 to more than 5000 dollars with 5 to 7 activity restricted days [1-4]. Meningitis clinically manifests as photophobia, nuchal rigidity, altered mental status, fever, and headache. Unlike bacterial meningitis, aseptic meningitis has a self-limiting course without specific treatment.

Primary HSV has been increasingly recognized as a cause of viral meningitis in adults. In contrast to HSV encephalitis, which is almost exclusively due to HSV-1, viral meningitis in immunocompetent adults is generally caused by HSV-2. In a study of 144 patients with aseptic meningitis, HSV-2 was the second most common etiology (17\%) after enteroviruses (26\%) [5]. Between 13 and 36 percent of patients presenting with primary genital herpes have clinical findings consistent with meningeal involvement, including headache, photophobia and meningismus. On the other hand, genital lesions are present in approximately 85 percent of patients with primary HSV-2 meningitis and generally precede the onset of CNS symptoms by seven days. The CSF profile includes a pleocytosis with a predominance of lymphocytes, and a normal CSF glucose concentration. HSV meningitis can also occur without evidence of genital lesions, although this is less common.

There is no standard approach to the treatment of HSV meningitis [6]. For hospitalized patients, we prefer intravenous Acyclovir at $10 \mathrm{mg} / \mathrm{kg}$ administered every eight hours. Patients can be switched to an oral agent on discharge for a total of 10 to 14 days of treatment.

Patients typically present with evidence of meningeal inflammation and cerebrospinal fluid with negative routine bacterial cultures. Gram staining of CSF is negative for bacteria. On CSF analysis, WBC count is usually found to be less than 250 cells $/ \mu \mathrm{L}$ with predominance of lymphocytes. Protein is slightly elevated and glucose concentration is within normal limits [1].

In CSF (healthy or infected), there is normally no plasma cell pleocytosis. However, its presence in CSF has been documented in multiple myeloma, multiple sclerosis, leukemia, and lymphoma. It has also been found in some patients with West Nile Virus encephalitis [2], but not in meningitis. This particular patient is one of the first documented cases with CSF plasma cell pleocytosis in aseptic meningitis infection.

\section{CASE REPORT}

A 36-year-old morbidly obese, Hispanic male presented with severe constant throbbing headache associated with fever, cough and backache which was preceded by pharyngitis 3 days prior to the presentation. Headache was exacerbated by cough but patient denied nuchal rigidity, nausea, vomiting, photophobia, rashes, or any sick contacts. Past medical history is significant for asthma and obesity. His home medications included Albuterol in- 
haler for asthma, Augmentin for pharyngitis, and Tylenol for headache and fever. The patient lives with his spouse and is sexually active. He denied any alcohol or illicit substance abuse. Family history is significant for diabetes and renal disease in his mother. He is $175 \mathrm{~cm}$ tall, weighs $123.4 \mathrm{~kg}$ with BMI of 40.3. Upon presentation, he had temperature of $100.3 \mathrm{~F}$ and had respiratory rate of about 20 breaths/min. All other vital signs were within normal limits. Subsequent hemogram and metabolic panel including creatinine and electrolytes were unremarkable. Shortly after admission, fever spiked at 102.9 and the patient became mildly tachycardic (102 beats/min). On physical exam, he was alert and oriented without any focal neurological deficits. He had mild pharyngeal erythema and varicose veins. He had no rashes. At this point, the differential diagnoses included meningitis (aseptic and bacterial), encephalitis, hydrocephalus, aneurysm, and ICH. Intervention/Response to Treatment: In the Emergency Department, the patient was given empiric antibiotics (Ceftriaxone, Vancomycin, and Ampicillin) due to clinical suspicion of bacterial meningitis. Head CT scan and Lumbar puncture were performed. CT scan yielded a normal study. CSF cytology showed leukocytosis $\left(825\right.$ cells $\left./ \mathrm{mm}^{3}\right)$ with lymphocyte predominance $(88 \%)$ and plasma cell pleocytosis $(7 \%-12 \%)$, high protein concentration $(90 \mathrm{mg} / \mathrm{dL})$, normal glucose $(52 \mathrm{mg} / \mathrm{dL})$, and unremarkable Gram stain. PCR analysis was negative for HSV-1 and positive for HSV-2. The antibiotic regimen was discontinued, as these results were highly suggestive of viral meningitis due to HSV-2 infection. The patient was admitted to Medicine Service for further management. He was given Tylenol\#3 for headache. On Day 2, the patient was afebrile (98.7 F) and his temperature was stable throughout the rest of the hospital course. CSF culture yielded no growth and was non-reactive to VDRL. The patient was also seen by Infectious Disease Service and was subsequently discharged on Day 5 with Valaciclovir $2 \mathrm{~g}$ for 10 days and Tylenol No. 3. After completing treatment, the patient was seen in the Medicine Ambulatory Clinic, when he reported feeling healthy. Subsequent follow up was unremarkable.

\section{DISCUSSION}

Viral Meningitis should be suspected in those with CSF findings of cell count $<500 / \mathrm{microL},>50$ percent CSF lymphocytes, protein concentration less than 80 to 100 $\mathrm{mg} / \mathrm{dL}$, normal glucose concentration, and negative Gram stain. Plasma cell pleocytosis in CSF has been document- ed in some disease processes including West Nile encephalitis and Multiple Sclerosis, but this is one of the first cases of plasma cells associated with HSV-2 Meningitis.

Any case of suspected meningitis should be treated with empiric antibiotics, especially elderly or immunocompromised patients who should be received empiric antibiotics for $48 \mathrm{hrs}$. Careful history including medications, exposure to ticks, mosquitoes, travel history, and TB exposure should be obtained. Blood and CSF cultures should be preferably obtained before initiating antibiotics. Lumbar Puncture plays an important role in diagnosing the etiology. Aseptic or Viral Meningitis usually resolves spontaneously with symptomatic treatment and antibiotics should be discontinued once CSF results are suggestive of viral etiology for meningitis.

PCR analysis revealed HSV-2 infection which is known to be associated with meningitis. The patients with HSV2 meningitis should also be screened for other STDs including syphilis and HIV. If HSV-2 meningitis is suspected in a patient with concomitant genital lesions, acyclovir (10 mg/kg IV q8) can be initiated empirically.

\section{REFERENCES}

[1] Johnson, R.J. (2011) Aseptic meningitis in adults. In: UpToDate, Basow, D.S., Ed., UpToDate, Waltham.

[2] Carson, P.J., Steidler, T., Patron, R., Tate, J.M., Tight, R., Smego Jr., R.A. (2003) Plasma cell pleocytosis in CSF in patients with West Nile Virus encephalitis. Clinical Infectious Diseases, 37, e12-e15. http://dx.doi.org/10.1086/375692

[3] Cohen, J.I. (2011) Chapter 191. Enteroviruses and reoviruses. In: Longo, D.L., Fauci, A.S., Kasper, D.L., Hauser, S.L., Jameson, J.L., Loscalzo, J., Eds., Harrison's Principles of Internal Medicine, 18e.

[4] Parasuraman, T.V., Frenia, K. and Romero, J. (2001) Enteroviral meningitis. Cost of illness and considerations for the economic evaluation of potential therapies. Pharmacoeconomics, 19, 3-12.

http://dx.doi.org/10.2165/00019053-200119010-00001

[5] Kupila, L., Vuorinem, T., Vainionpaa, R., Hukkanen, V., Marttila, R.J. and Kotilainen, P. (2006) Etiology of aseptic meningitis and encephalitis in an adult population. Neurology, 66, 75-80. http://dx.doi.org/10.1212/01.wnl.0000191407.81333.00

[6] Landry, M.L., Greenwold, J. and Vikram, H.R. (2009) Herpes simplex type-2 meningitis: Presentation and lack of standardized therapy. American Journal of Medicine, 122, 688-689.

http://dx.doi.org/10.1016/j.amjmed.2009.02.017 